University of Nebraska - Lincoln

DigitalCommons@University of Nebraska - Lincoln

2020

\title{
Vertical-Surface Navigation in the Neotropical Whip Spider Paraphrynus laevifrons (Arachnida: Amblypygi)
}

Patrick Casto

Daniel D. Wiegmann

Vincent J. Coppola

Daniele Nardi

Eileen A. Hebets

See next page for additional authors

Follow this and additional works at: https://digitalcommons.unl.edu/bioscihebets

Part of the Animal Sciences Commons, Behavior and Ethology Commons, Biology Commons, Entomology Commons, and the Genetics and Genomics Commons

This Article is brought to you for free and open access by the Papers in the Biological Sciences at DigitalCommons@University of Nebraska - Lincoln. It has been accepted for inclusion in Eileen Hebets Publications by an authorized administrator of DigitalCommons@University of Nebraska - Lincoln. 


\section{Authors}

Patrick Casto, Daniel D. Wiegmann, Vincent J. Coppola, Daniele Nardi, Eileen A. Hebets, and Verner P. Bingman 


\title{
Vertical-Surface Navigation in the Neotropical Whip Spider Paraphrynus laevifrons (Arachnida: Amblypygi)
}

\author{
Patrick Casto, ${ }^{1,2}$ Daniel D. Wiegmann, ${ }^{1,2}$ Vincent J. Coppola, ${ }^{4}$ \\ Daniele Nardi, ${ }^{5}$ Eileen A. Hebets, ${ }^{6}$ and Verner P. Bingman ${ }^{2,3}$
}

1. Department of Biological Sciences, Bowling Green State University, Bowling Green, Ohio, USA

2. J. P. Scott Center for Neuroscience, Mind and Behavior, Bowling Green State University, Bowling Green, Ohio, USA

3. Department of Psychology, Bowling Green State University, Bowling Green, Ohio, USA

4. Department of Behavioral Sciences, University of Findlay, Findlay, Ohio, USA

5. Department of Psychological Science, Ball State University, Muncie, Indiana, USA

6. School of Biological Sciences, University of Nebraska-Lincoln, Lincoln, Nebraska, USA

Corresponding author - Patrick Casto, Department of Biological Sciences, Bowling Green State University, Bowling Green, OH, 43403, email pcasto@bgsu.edu

\begin{abstract}
Studies on whip spider navigation have focused on their ability to locate goal locations in the horizontal plane (e.g., when moving along the ground). However, many species of tropical whip spiders reside and move along surfaces in the vertical plane (e.g., trees). Under controlled laboratory conditions, the current study investigated the ability of the tropical whip spider, Paraphrynus laevifrons, to return to a home shelter on a vertical surface in the presence of numerous, similar, and competing refuge sites, as well as the distribution of navigational errors in the vertical, horizontal, and diagonal plane. We also assessed the relative importance of sensory cues originating from a previously occupied home shelter compared to the position of a previously occupied shelter in guiding shelter choice. It was found that $P$. laevifrons displays robust fidelity in relocating a home shelter on a vertical surface. When navigational errors did occur, they were not significantly different in all three directions. Additionally, cue-conflict test trials revealed that cues associated with an original home shelter,
\end{abstract}


likely self-deposited chemical signals, were more important than sources of positional information in guiding the shelter choice of $P$. laevifrons.

Keywords: arthropod, chemoreception, olfaction, spatial navigation, vertical information

\section{Introduction}

Navigational behavior has been extensively studied in a number of arthropod taxa including desert ants, fiddler crabs, bees, and spiders (reviewed by Cheng 2012). Many of the terrestrial arthropods that have been studied inhabit two dimensional or slightly cluttered environments and use sensory-specific navigational strategies. For example, the desert ant, Cataglyphis fortis, and Australian ant, Melophorus bagoti, inhabit mostly flat, ground surfaces with little clutter and canopy (Cheng et al. 2012). In this type of habitat, arthropod navigation is often guided by path integration (Wehner 2003) and goal-oriented movements with respect to stimuli found in the environment (e.g., landmark following [Wehner 2003; Collett 2010], including olfactory landmarks [Steck et al. 2009; Buehlmann et al. 2015], trails of odors laid on the ground [Hölldobler and Wilson 1990], and panoramic scenes [Graham and Cheng 2009]).

As noted above, most of the literature on arthropod navigation-and animals in general-focuses on movements on a horizontal plane. Research on navigation in threedimensional space is relatively scarce and has only recently been given attention (Rheinlaender et al. 2007; for reviews see Davis et al. 2018; Jeffery et al. 2013, 2015). Particularly interesting is the comparison between the spatial encoding of vertical and horizontal dimensions. In terms of goal-oriented behavior, the accuracy and rate of learning the location of a goal indicate that the representation of horizontal and vertical space can be influenced by style of locomotion (Davis et al. 2018); i.e., surface-bound, 2D movements (e.g., walking on a mound or a wall) compared to non-surface-bound, volumetric movements (e.g., in air or water).

Fishes, as non-surface-bound animals, show equal learning rate and accuracy when encoding the location of a goal in the vertical and horizontal plane (Holbrook and Perera $2009,2013)$. Some studies, however, have suggested that fishes display greater accuracy encoding the vertical position of a goal location (Davis et al. 2014). By contrast, flying animals-e.g., bees (Nieh et al. 2003), bats (Sumiya et al. 2017), and hummingbirds (Hurly et al. 2010) - are characterized by no consistent pattern in favoring the representation of a goal location in the vertical and horizontal planes.

Conversely, it seems that surface-bound animals display a more accurate encoding of the horizontal component of a goal location. For example, in Jovalekic et al. (2011), rats tested in a vertically extended pegboard or a cubic maze exhibited a horizontal layer foraging strategy and temporal discounting of effort (they preferred to take an easier, shallow path subcomponent first rather than a steeper path). This pattern of behavior has been recently replicated (Grieves et al. 2020; see also Grobéty and Schenk 1992). In rats, the distinction between the representation of horizontal and vertical space is also apparent in the spatial response properties of hippocampal system neurons (Grieves et al. 2020; Hayman et al. 2011; for a contrast with bats see Ulanovsky 2011; Yartsev and Ulanovsky 2013; for a review see Jeffery et al. 2015). An analogous behavioral prioritization of the encoding of 
horizontal information has been shown in studies of desert ants (Ronacher 2020), dogs (Brandt and Dieterich 2013), and humans (Zwergal et al. 2016; but see Nardi et al. 2011; for a review see Davis et al. 2018). Notable, however, is that these surface-bound animals are more active along the horizontal plane of their natural habitat compared to the vertical (they traverse extensive horizontal distances with only some vertical displacement). The question that then arises is whether the same pattern of privileged horizontal encoding would apply to a surface-bound species whose ecology and locomotion is similarly distributed on horizontal and vertical surfaces. For example, the bull ant, Myrmecia midas, often forages up trees and has been shown to use panoramic visual cues while on the vertical surface of the tree to orient toward the nest location (Freas et al. 2018).

Amblypygi, commonly called whip spiders, are nocturnally active arachnids that live in tropical and subtropical habitats around the world, and they are exceptional navigators (Beck and Görke 1974; Hebets et al. 2014a, b; Bingman et al. 2017). Tropical whip spiders, in particular, routinely move on the vertical surface of their home tree as well as successfully navigate back to their home trees after they transition to moving on the ground. Navigation is enabled by the first pair of legs on whip spiders, called antenniform legs, which are not used for walking but rather are elongated, thin, and covered in various sensory sensilla. Whip spiders use the antenniform legs to detect environmental stimuli, particularly chemical cues, where the apical annuli bear unique olfactory and contact chemoreceptors (Hebets and Chapman 2000; Foelix and Hebets 2001). In the context of locating a home refuge, Wiegmann et al. (2019) demonstrated in a laboratory experiment that Phrynus marginemaculatus can learn to associate a home shelter with an artificial odor. Additionally, P. marginemaculatus can use self-derived chemical cues for shelter recognition (Casto et al. 2019). Tactile cues may also provide information for shelter recognition in P. marginemaculatus (Santer and Hebets 2009). Olfaction also appears to be necessary for longer-distance navigation by whip spiders that inhabit Neotropical forests (Beck and Görke 1974; Hebets et al. 2014a, b; Bingman et al. 2017).

These studies cited examined the ability of whip spiders to locate a goal solely in the horizontal plane. But as noted, many species of tropical whip spiders reside and move along vertically extended surfaces; e.g., trees (Phrynus pseudoparvulus, Hebets 2002; Heterophrynus batesii, Chapin 2014), rocky outcroppings, cave walls (Damon diadema, Weygoldt 2000; Phrynus longipes, Chapin 2015; Charinus asturius, Segovia et al. 2018), and cliff banks (Paraphrynus laevifrons, Corey and Hebets 2017). Indeed, the claws on their walking legs allow them to hold on to various rough surfaces and even move anterior/prosoma-down on a vertical substrate (Weygoldt 2000). Surprisingly then, to the best of our knowledge, there has been only one study that has only indirectly looked at their ability to locate a goal on a vertical surface. Hebets et al. (2014a, b) displaced whip spiders, P. pseudoparvulus, which had recently exited their daytime refuges, to the opposite side of the home tree. Those animals successfully returned to their home refuges, but importantly, the animals were deposited at the same height of the home shelter, i.e., they were not vertically displaced.

The current study aimed to investigate how whip spiders navigate to a shelter on a vertical surface and identify potential sensory mechanisms that may guide such vertical navigation. First, we tested the hypothesis that $P$. laevifrons are efficient vertical navigators, 
predicting they would display fidelity to a refuge on a vertical surface after a night of wandering when multiple, similar, and competing shelters are available. Because P. laevifrons typically moves fluidly on vertical surfaces in their tropical habitat (Corey and Hebets 2017), we also predicted that navigational errors would be similarly distributed between the horizontal and vertical dimensions; in other words, we did not expect better encoding of horizontal information compared to vertical. To explore the sensory control of verticalsurface navigation, we additionally carried out test trials to assess the relative importance of sensory cues originating from a previously occupied home shelter compared to the position of a previously occupied shelter in guiding shelter choice. Given the general importance of olfactory cues guiding movements on the horizontal plane (Beck and Görke 1974; Bingman et al. 2017; Wiegmann et al. 2019) and the known ability of whip spiders to recognize a home shelter using self-deposited chemical cues (Casto et al. 2019), we predicted that subjects would prefer to return to a previously occupied shelter that was moved (correct olfactory cues; incorrect positional cues) over a nonpreviously occupied shelter that was in the correct location (incorrect olfactory cues; correct positional cues).

\section{Methods}

\section{Subjects}

Twelve P. laevifrons were used; three were hatched in captivity at Bowling Green State University and nine were collected from Las Cruces Biological Station in Puntarenas, Costa Rica (Permit Number 060-DGVS-2016). Prior to testing, the three animals hatched in captivity were housed together in a $91 \times 45.5 \times 43 \mathrm{~cm}$ tank, while the nine animals collected in Costa Rica were housed individually in $30 \times 19 \times 20 \mathrm{~cm}$ containers. The containers were filled with coconut fiber substrate, which holds moisture well and nourishes springtails for cleaning the substrate, and various sizes of vertically oriented cork bark for shelter. The whip spiders were fed crickets twice weekly, provided water ad lib, and sprayed with distilled water daily for hydration. The housing room was lit by overhead broad-spectrum fluorescent lights (400-750 nm) set on a 12:12 h light-dark cycle (19:00-7:00 dark phase); temperature was maintained between 27 and $29^{\circ} \mathrm{C}$ and humidity was kept between 65 and $70 \%$.

\section{Experimental environment}

The room in which the experiment was conducted was climate-controlled similar to the housing room (see above). The experimental apparatus consisted of a white, acrylic, vertically oriented shelter board (VSB) measuring $50 \times 50 \times 1 \mathrm{~cm}$ with a $20 \times 50 \times 1 \mathrm{~cm}$ white acrylic base (Fig. 1). Nine $4 \times 1 \mathrm{~cm}$ shelter entrances were cut into the VSB in a three-bythree grid pattern (Fig. 1a). The shelters, $8 \times 4 \times 6 \mathrm{~cm}$ black acrylic containers with hookside Velcro placed on their floors, were attached to the back surface of the VSB (Fig. 1b). Shelters were spaced such that the vertical and horizontal edges of the shelters were $9 \mathrm{~cm}$ from the parallel edge of the adjacent shelters. The distance from the edges of the perimeter outermost shelters to the top and bottom edges of the VSB was $12 \mathrm{~cm}$, while the distance from the corner edges of the diagonal outermost shelters and from the left and right corner 
edges of the VSB was $10 \mathrm{~cm}$. Notable, when extending its antenniform legs while stationary on the VSB, a whip spider could touch only one shelter entrance.

a

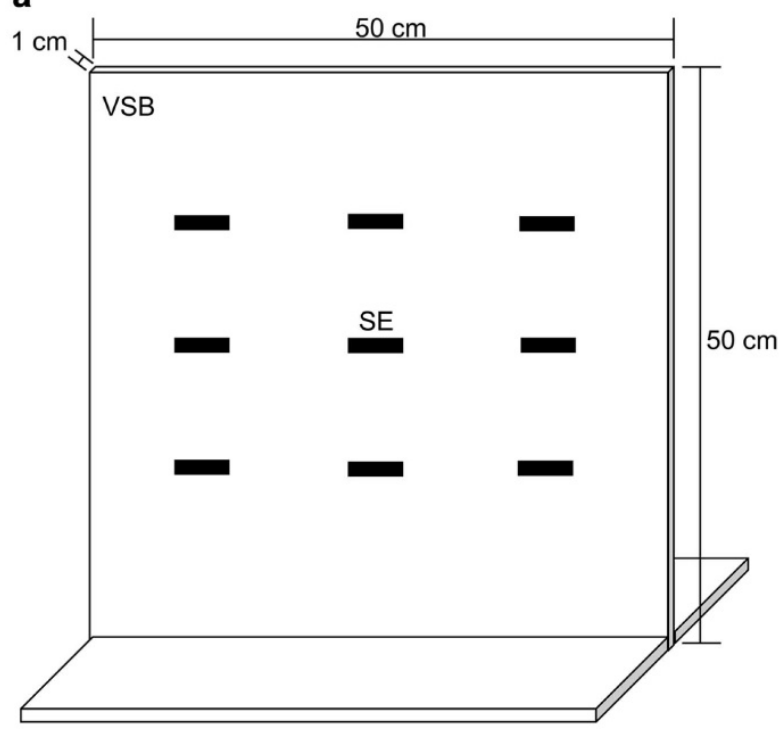

b

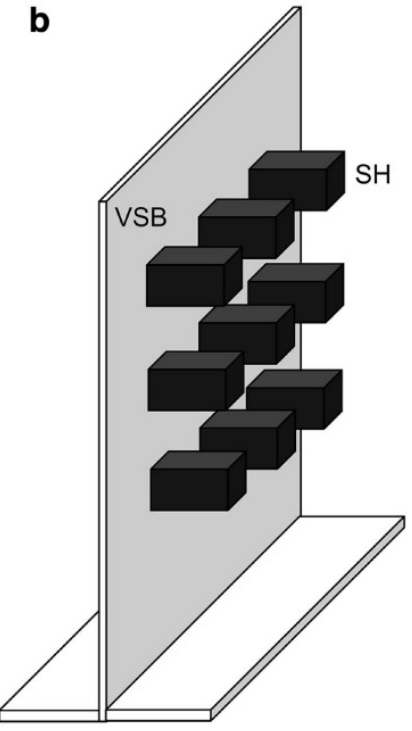

Figure 1. Schematic of the vertical shelter board (VSB). (a) Front view showing the nine shelter entrances (SE), each measuring $4 \times 1 \mathrm{~cm}$ and spaced $9 \mathrm{~cm}$ from the parallel edge of adjacent shelters, which are arranged in a $3 \times 3$-grid pattern. (b) Back view showing the nine black acrylic shelters $(\mathrm{SH})$, each $8 \times 4 \times 6 \mathrm{~cm}$.

The VSB base was placed adjacent to one wall of a $100 \times 100 \times 20 \mathrm{~cm}$ enclosure arena with clear acrylic plastic walls and a white acrylic plastic floor. This enclosure, containing the VSB, was positioned at the center of a wooden scaffolding structure, from which a video camera (Swann Alpha Series) was mounted $10 \mathrm{~cm}$ from the edge of the arena opposite the VSB and $25 \mathrm{~cm}$ above the floor of the arena. For 10 of the 12 subjects, the camera recorded video (stored on a Swann Alpha Series DVR) for a period of $13 \mathrm{~h}$, beginning 30 min before lights off and ending $30 \mathrm{~min}$ after lights on, thus requiring two Univivi Array Infrared Illuminator lights (placed on the wooden scaffold approximately $55 \mathrm{~cm}$ above the enclosure arena) to allow for video recording in the dark. Whip spiders are unlikely to see in the infrared (Wiegmann, unpublished data); therefore, we assumed that subjects could not use visual cues while navigating during the night. Positional information was extracted from the recordings, which were used to assess the locations of a subject on the VSB, whether an animal left the VSB during the night, and how many times a subject exited and entered a shelter during a night.

\section{Experimental procedures}

Subjects experienced 3 multi-trial phases of experimentation; whereas Phase I $(N=12)$ consisted of 3 trials, Phases II $(N=11)$ and III $(N=9)$ each consisted of 4 trials. Subjects proceeded from trial to trial (and phase to phase) only after successfully completing the current 
trial (or phase), which was defined as an animal leaving a shelter after lights off and returning to a shelter before lights on.

\section{Phase I: orientation trials}

Three Phase I (orientation) trials were conducted in order to (i) familiarize subjects with the VSB, (ii) establish a starting home shelter (i.e., forced shelter fidelity), and (iii) give subjects experience with being nudged (gently, with a paint brush) from their shelters (important for Phase III). During Phase I, only one shelter was accessible (always at the center of the $3 \times 3$ grid) while the shelter entrances to all other shelters were blocked off on the back side with poster board. A $2 \times 3 \mathrm{~cm}$ rectangular sponge, dampened as necessary with reverse osmosis water, was placed in the shelter for drinking and humidity.

During the first and third trials of Phase I, subjects were required to leave the shelter on their own after lights off. On trial 2, subjects were blocked in the shelter until being nudged out of the shelter with a paint brush at 90 min after lights off (20:30) but were otherwise undisturbed during the trial. For all trials, if an animal did not return during the night (before lights on), then it was placed back in the center shelter and the trial was repeated. Criterion to move on to Phase II was successful completion of each of the three Phase I trials. Subjects took an average of $3.90 \pm 0.37$ trials to complete Phase I.

\section{Phase II: homing trials}

During Phase II, all nine shelters, each containing a $2 \times 3 \mathrm{~cm}$ dampened sponge, were made accessible with the intent of testing shelter fidelity. Shelter fidelity was defined as leaving a shelter (and perhaps even the VSB) after lights off and then returning to the same shelter before lights on. Thus, if a subject returned to the shelter it occupied at the beginning of the trial, the choice was considered correct. By contrast, if the subject chose any of the other eight shelters, the choice was considered incorrect. Furthermore, vertical component errors (i.e., when a subject returned to a shelter that was either above or below the original shelter), horizontal component errors (i.e., when a subject returned to a shelter that was either to the left or right of the original shelter), and diagonal component errors (i.e., when a subject chose any other shelter not defined as vertical or horizontal) were recorded. If a subject did not choose a shelter by lights on, the trial was considered unsuccessful, the subject was nudged back to the shelter occupied at the beginning of the trial, and the trial repeated.

Importantly, although all subjects began the first trial of Phase II in the center shelter (trained to during Phase I), the correct shelter on all subsequent trials was dependent upon which shelter they returned to the night before (i.e., the correct shelter could have varied from trial to trial, depending on which shelter the subject chose at the end of the night). Thus, shelter choice errors could be one or two units away from the correct shelter whenever the correct shelter was not in the center. No distinction was made between errors that were made one or two units away. Finally, trials were additionally categorized as $\mathrm{On}$ trials if the subject remained the entire night on the VSB or Off trials if it ventured off the VSB onto the surrounding floor. 
Phase III: cue conflict

The cue conflict trials were designed to assess the relative importance of sensory information associated with the physical shelter occupied the day before, likely self-deposited sensory cues (Casto et al. 2019), and positional information (e.g., path integration, or visual cues, associated with the location of the shelter occupied the day before). Subjects, initially blocked in their home shelters from the day before, were nudged out of their home shelters with a paint brush one hour after lights off (20:00), at which point their home shelter was switched with an adjacent shelter from the same row (if the home shelter was in the middle column, a coin flip determined whether the shelter was switched with the adjacent left or right shelter). This switching of the home shelter with an adjacent shelter sets the sensory cues emanating from the home shelter itself in conflict with the positional information from path integration and/or the surrounding environment. Otherwise, all shelter entrances were essentially identical, minimizing the likelihood that tactile cues could have been used for shelter discrimination.

Note that the shelter occupied at the beginning of the trial will henceforth be referred to as the original home shelter even after being moved to a new location, while the location the home shelter originally occupied at the beginning of the night (which is now occupied by a new clean shelter) will henceforth be termed original shelter location. As with Phase II trials, all nine shelters were continually accessible, and choices to either the original home shelter or the original shelter location were considered correct (important for data analysis). If at the end of the night a subject chose a shelter that was not the original home shelter, then the original home shelter was replaced with a clean shelter, and the shelter that the subject chose then became the home shelter for the following trial. Finally, if a subject did not choose a shelter by lights on, then the original home shelter was returned to its original location, the subject was placed back in the original home shelter, and the trial was repeated.

\section{Data analyses}

Of the 12 subjects tested, one did not leave the shelter for any Phase II trial; thus, the Phase II analyses are based on $N=11,9$ of whom were video recorded. For Phase II data, a onesample $t$-test was used to compare the percent correct choices (On and Off trials combined), again defined as returning to the shelter where the subjects began the night, to random chance $(11.11 \%$ with nine available shelters). Two one-sample $t$-tests were then used to compare the percent correct choices to random chance for the On and Off trials separately. A chi-squared test was used to compare whether leaving the VSB had an impact on homing accuracy (note that a $t$-test could not be used for this analysis, as some subjects either never or always left the VSB on all 4 trials). To assess the potential directional biases in the distribution of errors in Phase II, we tallied the number of errors above or below a starting location (considering only trials starting in the middle row of the $3 \times 3$ grid) as well as the number errors to the left and right of a starting location (considering only trials starting in the middle column of the $3 \times 3$ grid). Finally, a repeated measured ANOVA was used to assess differences in error type (vertical, horizontal, and diagonal; see above). Note that subjects that did not make a single error $(N=4)$ were excluded from this analysis.

Of the 12 subjects, only 9 were available for Phase III trials; thus, the Phase III analyses are based on $N=9$. A one-sample $t$-test was used to compare the percent of correct choices, 
defined as a choice to either the original home shelter or the original shelter location, to random chance performance (chance is now $22.22 \%$, as two of the nine shelters were considered correct choices), and a paired-samples $t$-test was used to assess the difference between the percent correct choices to the original home shelter compared to the original shelter location. Finally, we used a paired-samples $t$-test to compare how often subjects switched shelters throughout the night between Phase II and Phase III. All of these analyses were performed using SPSS Statistics for Windows, Version 25.0, and the criterion for significance was $p<0.05$.

\section{Results}

Phase II trials were conducted to determine if subjects returned to the shelter that they began in the night before. On average, $56.82 \%(S E=11.72 \%)$ of choices were to the shelter where the subject began the night, and this was significantly above chance $(11.11 \%), t(10)=3.90$, $p<0.01$. Based on the 9 subjects that were video recorded, correct choices did not depend on whether the animal left the VSB (Off trials; $M=52.00 \%, S E=15.26 \%$ ) or not (On trials; $M=61.86 \%, S E=17.26 \%$ ). Subjects chose the shelter that they began the night in above chance $(11.11 \%$ ) on both Off trials, $t(6)=2.75, p<0.05$, and On trials, $t(7)=2.68, p<0.05$ (Fig. 2a). Furthermore, there was no statistical effect of trial type (Off vs. On) on total correct choices to shelters in which the subject began the night (Off-Correct $=9$, Off-Incorrect $=5$, On-Correct $=13$, On-Incorrect $=9), \chi^{2}(1)=0.10, p=0.76$.
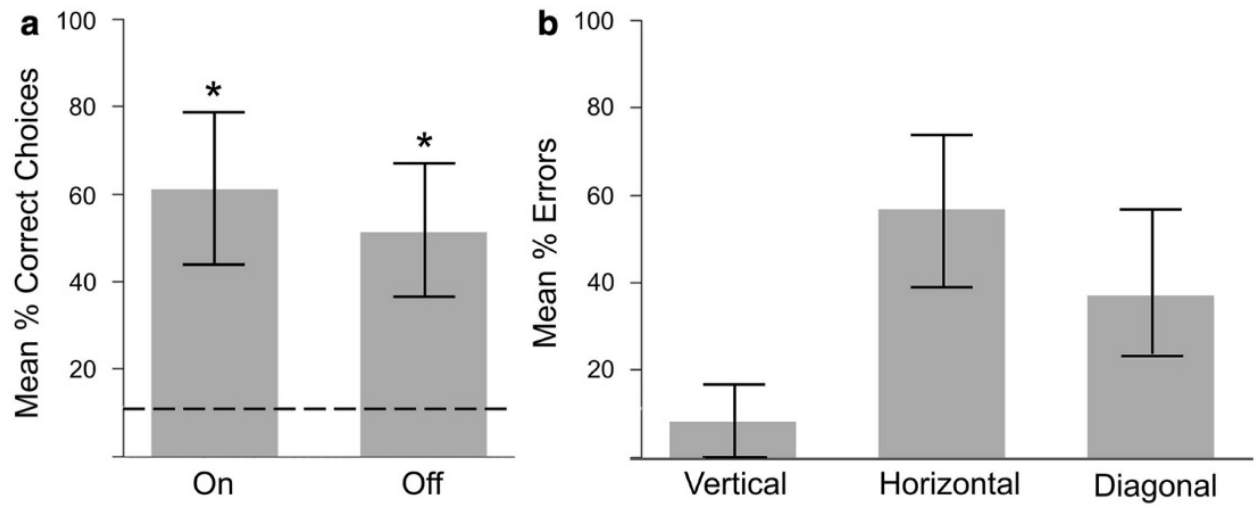

Figure 2. (a) Mean $( \pm$ SEM) percent correct choices of Phase II trials in which the animal did not (On) and did (Off) leave the VSB. Note that mean percent correct was significantly better than chance $(11.11 \%$, indicated by dashed line) regardless of whether or not the animal left the VSB. (b) Mean ( \pm SEM) percent of errors made in Phase II across the three error types (vertical, horizontal, and diagonal).

Another goal of Phase II was to assess the distribution of errors and determine if, when committing errors, a subject would be more likely to make a vertical, horizontal, or diagonal error with respect to the shelter occupied when the night began. It was found that subjects made 4 errors below the correct shelter while making 1 error above the correct shelter 
in the vertical plane, whereas subjects made 4 left and 5 right errors in the horizontal plane. These numbers were too small for any meaningful statistical analysis. A repeated measures ANOVA found no significant difference in the type of error committed, $F(2,12)=2.47$, $p<0.13$ (Fig. 2b). Potentially interesting is that of the 11 errors committed when the correct shelter was not the center shelter, 3 errors were made to the center shelter.

In Phase III, when sensory cues emanating from the home shelter were put in conflict with the positional cues from the surrounding environment, the mean percent of choices made to one of the two correct shelters (i.e., the original home shelter or original location) was $52.78 \%(S E=6.51 \%)$, which was significantly greater than chance $(22.22 \%), t(8)=4.69$, $p<0.01$ (Fig. 3). When the two correct choices were compared, the mean percent of correct choices to the original home shelter $(M=77.78 \%, S E=12.44 \%)$ was significantly greater than the mean percent of choices to the original shelter location $(M=22.22 \%, S E=12.44 \%)$, $t(8)=2.23, p=0.05$ (Fig. 3) .

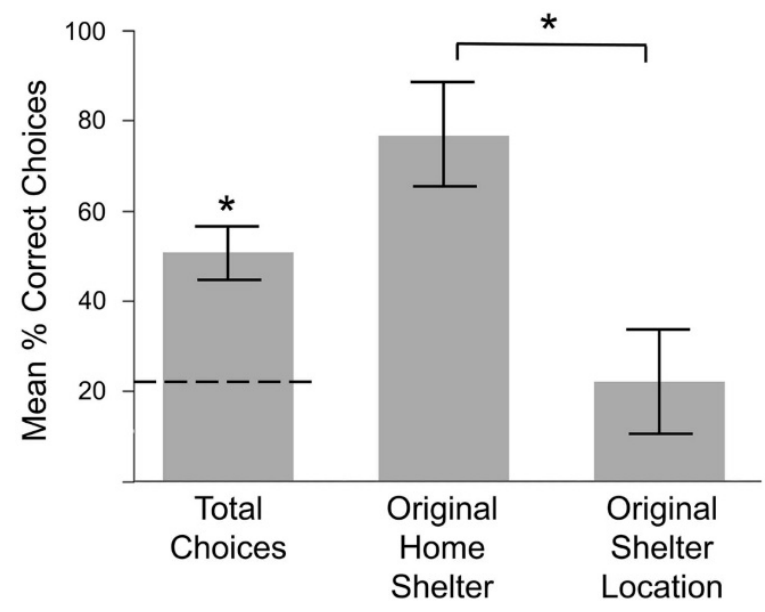

Figure 3. Mean ( \pm SEM) percent correct of all choices made in Phase III (cue conflict), as well as the breakdown of the percent of correct choices made to the moved location (original home shelter) and to the original shelter location. Note that the mean percent of correct choices made in Phase III was significantly greater than chance (22.22\%, dashed line). However, when correct choices were divided into original home shelter or original shelter location, the percent of correct trials in which subjects chose the original home shelter was significantly greater than to the original shelter location. $\left({ }^{*} p \leq 0.05\right)$

Of potential interest is the degree to which subjects may have sampled the ostensibly identical shelters before settling in one, in part to assess how motivated they were in returning to their home shelter. For Phase II, subjects on average made $5.17 \pm 0.86$ entrances into shelters during a night, while during Phase III, subjects made $3.78 \pm 0.63$ entrances into any shelters. There was no difference between the mean number of shelter entrances made in Phase II and III, $t(7)=0.51, p=0.63$. The multiple entrances that characterize a night suggest that errors were not substantially due to low motivation. 


\section{Discussion}

Our results demonstrate that the whip spider Paraphrynus laevifrons reliably returns to a home shelter on a vertical surface in the presence of numerous alternative shelter sites, a result which builds on field observations showing refuge site fidelity on home trees even after experimental, horizontal displacements on the home tree (Hebets 2002; Hebets et al. 2014a). The robustness of the observed vertical navigational ability is highlighted by the finding that even when leaving the vertical shelter board and wandering on the floor of the arena, subjects still reliably returned to their original shelter. The vertical navigational ability of tropical whip spiders, in this case Phrynus pseudoparvulus (previously identified as Phrynus parvulus, see de Armas and Viquez 2001), can be inferred from their behavior in the wild (Hebets 2002; Hebets et al. 2014a), but the current study is the first to document this ability experimentally.

The observed accuracy for homing on the VSB in the current study supports the hypothesis (Davis et al. 2018) that species movement ecology influences the properties of spatial representations in horizontal and vertical space. When errors were made in homing, they were not statistically different in any of the three directions (Fig. 2b). Although no clear pattern emerged, when considering studies that have assessed the encoding accuracy of vertical and horizontal information in 3D navigation, a possible distinction that has been proposed is based on species locomotion style (Davis et al. 2018). Compared to nonsurface-bound animals that move in volumetric spaces, surface-bound animals seem to be less skilled at encoding the vertical component of a goal location, possibly because they are anchored to a substrate and have fewer degrees of freedom of movement (Davis et al. 2018). However, as noted in the Introduction, most of the surface-bound animals tested to date travel mainly in the horizontal plane of their natural habitat because their resources are unreliable and scarce in the vertical dimension (e.g., Nieh et al. 2003). In this context, our study provides meaningful insights because whip spider movements seem to be as likely to occur on a vertical or horizontal surface. For example, based on casual observations in the field, movements on the vertical surface of the home tree are as frequent as horizontal movements on the ground. In our study, we found that the frequency of vertical errors was not significantly different from that of horizontal or diagonal errors. Numerically, but not significantly, vertical errors were the fewest among the types of errors (Fig. $2 b)$. Furthermore, when diagonal errors were excluded from the analysis, a paired samples $t$-test showed that horizontal errors were more likely to occur than vertical errors $(p<0.03$, see also Fig. 2b). Thus, the possibility of more accurate navigation in the vertical plane in $P$. laevifrons warrants further investigation.

The successful navigation in the vertical plane raises the question of how $P$. laevifrons may sense movements in that plane given the importance of gravity. Whip spiders and other arachnids have mechanical stress-sensing organs on their legs, called slit-sensilla, or lyriform organs when clustered into dense groups (Barth and Stagl 1976). These stresssensing organs might enable sensing differences in vertical vs. horizontal movements; gravity has a greater effect on leg stress when the spider, Cupiennius salei, adopts a vertical compared to a horizontal body orientation (Barth 2002). Indeed, the body orientation of an organism may have an important role in the ability to perceive and discriminate different 
geometric planes. For example, Casali et al. (2019) observed grid-cell odometry in the brains of rats that were able to walk on a vertical surface with a vertically oriented body axis; a behavioral procedure that contrasted with Hayman et al. (2011) who studied rats moving horizontally on a pegboard. It is still uncertain if whip spiders can use the lyriform organs to extract navigational information while moving on a vertical surface.

The Phase III results revealed that some signal associated with a shelter previously occupied by a whip spider has more control over the final shelter choice than the location of the shelter occupied the previous day. Our experiment was not designed to specify what that intrinsic signal may have been, but we are comfortable proposing that it was olfactory in nature. Olfaction has consistently been observed to be crucially important for the navigational behavior of whip spiders (Beck and Görke 1974; Hebets et al. 2014a, b; Bingman et al. 2017; Casto et al. 2019; Wiegmann et al. 2019). Indeed, following displacement on the home tree, Hebets et al. (2014b) observed a loss of navigational ability after impairment of the distal portions of the antenniform legs, which are the exclusive site of olfactory transduction. More importantly, Casto et al. (2019) demonstrated that trace chemical signals deposited by a whip spider itself could support the recognition of a home shelter.

Of potential interest is the degree to which subjects may have sampled the ostensibly identical shelters before settling in one, in part to assess how motivated they were in returning to their home shelter. Typically, multiple shelters were entered during a night's wandering. Hence, the subjects appeared motivated to sample shelters, perhaps in search of a recognition signal. Relevant here, however, is that females may be more site-faithful than males (Hebets 2002). Therefore, the possibility remains open that some of the errors recorded were a consequence of low motivation, which may have been more likely in our male subjects. Unfortunately, we were unable to sex the subjects of the current study (sexing $P$. laevifrons with certainty is difficult even after sacrificing the animals, which we wished to avoid).

In summary, this study was the first to investigate vertical navigational behavior in any whip spider species under controlled, laboratory conditions. We found that $P$. laevifrons exhibits fidelity to a refuge on a vertical surface, and when errors occurred, they were not statistically different in the vertical, horizontal and diagonal planes. Shelter choice was controlled more by signals associated with a previously occupied shelter than the location of a shelter when a night began, and we propose the signal to be a self-deposited chemical cue perceived by olfaction, although a contact chemosensory signal cannot be excluded. The robustness of $P$. laevifrons' navigational ability on a vertical surface advances our understanding of how animal navigation can be shaped by species movement ecology (Chapin and Hebets 2016; Davis et al. 2018), and in our view, motivates a more energetic comparative approach toward better understanding of how movement ecology can influence sensory guidance and spatial resolution in the context of navigation.

Acknowledgments - We are grateful for financial support from the National Geographic Society and the National Science Foundation (IOS 1457304). The data presented were collected by Meghan E. Moore and submitted in partial fulfillment for the requirements of a Master of Arts degree at Bowling Green State University. The datasets generated during and/or analyzed during the current study are available from the corresponding author on reasonable request. 
Authors' contributions - All authors contributed to the study: conceptualization-VPB, DDW; formal analysis-VPB, DDW, VJC; writing-PC, DDW, VJC, DN, EAH, VPB. All authors approved the final manuscript.

Conflicts of interest - The authors declare that they have no conflicts of interest.

\section{References}

Barth FG (2002) Proprioreception. In: Barth FG (ed) A Spider's World. Springer, Berlin, pp 113-127

Barth FG, Stagl J (1976) The slit sense organs of arachnids: a comparative study of their topography on the walking legs (Chelicerata, Arachnida). Zoomorphology 86:1-23

Beck L, Görke K (1974) Tagesperiodik, Revierverhalten und Beutefang der Geisselspinne Admetus pumilio CL Koch. Im: Freiland. Zeitschrift für Tierpsychologie 35(2):173-186. https://doi.org/ 10.1111/j.1439-0310.1974.tb00442.x

Bingman VP, Graving JM, Hebets EA, Wiegmann DD (2017) Importance of the antenniform legs, but not vision, for homing by the neotropical whip spider Paraphrynus laevifrons. J Exp Biol 220(5): 885-890. https://doi.org/10.1242/jeb.149823

Brandt T, Dieterich M (2013) "Right Door," wrong floor: a canine deficiency in navigation. Hippocampus 23(4):245

Buehlmann C, Graham P, Hansson BS, Knaden M (2015) Desert ants use olfactory scenes for navigation. Anim Behav 106:99-105. https://doi.org/10.1016/j.anbehav.2015.04.029

Casali G, Bush D, Jeffery K (2019) Altered neural odometry in the vertical dimension. Proc Natl Acad Sci USA 116(10):4631-4636. https://doi.org/10.1073/pnas.1811867116

Casto P, Gosser J, Wiegmann DD, Hebets EA, Bingman VP (2019) Self-derived chemical cues support home refuge recognition in the whip spider Phrynus marginemaculatus (Amblypygi: Phrynidae). J Arachnol 47(2):290. https://doi.org/10.1636/JoA-S-18-067

Chapin KJ (2014) Microhabitat and spatial complexity predict group size of the whip spider Heterophrynus batesii in Amazonian Ecuador. J Trop Ecol 30(02):173-177. https://doi.org/10.1017/S02664 67413000850

Chapin KJ (2015) Cave-epigean behavioral variation of the whip spider Phrynus longipes (Arachnida: Amblypygi) evidenced by activity, vigilance, aggression. J Arachnol 43(2):214-219. https://doi. org/10.1636/J15-04

Chapin KJ, Hebets EA (2016) The behavioral ecology of amblypygids. J of Arachnol 44(1):1-14. https:// doi.org/10.1636/V15-621

Cheng K (2012) Arthropod navigation: ants, bees, crabs, spiders finding their way. In: Zentall TR, Wasserman EA (eds) The Oxford handbook of comparative cognition. Oxford Univ Press, Oxford, pp 347-365

Cheng K, Middleton EJT, Wehner R (2012) Vector-based and landmark-guided navigation in desert ants of the same species inhabiting landmark-free and landmark-rich environments. J Exp Biol 215(18):3169-3174. https://doi.org/10.1242/jeb.070417

Collett M (2010) How desert ants use a visual landmark for guidance along a habitual route. Proc Natl Acad Sci USA 107(25):11638-11643. https://doi.org/10.1073/pnas.1001401107

Corey TB, Hebets EA (2017) Microhabitat use in the amblypygid Paraphrynus laevifrons. J Arachnol 45(2):223-230. https://doi.org/10.1636/JoA-S-16-0551

Davis VA, Holbrook RI, Burt de Perera T (2018) The influence of locomotory style on three-dimensional spatial learning. Anim Behav 142:39-47. https://doi.org/10.1016/j.anbehav.2018.06.002 
Davis VA, Holbrook RI, Schumacher S, Guilford T, Burt de Perera T (2014) Three-dimensional spatial cognition in a benthic fish Corydoras aeneus. Behav Proc 109(Pt B):151-156. https://doi.org/10.1016/ j.beproc.2014.08.011

de Armas LF, Víquez C (2001) Nueva especie de Phrynus (Amblypygi: Phrynidae) de Costa Rica Rica. Rev Ibér Aracnol 4:11-15

Foelix RF, Hebets E (2001) Sensory Biology of Whip Spiders (Arachnida, Amblypygi). Andrias 15: 129-140

Freas C, Wystrach A, Narendra A, Cheng K (2018) The view from the trees: Nocturnal bull ants, myrmecia midas, use the surrounding panorama while descending from trees. Front Psychol 9:16. https://doi.org/10.3389/fpsyg.2018.00016

Graham P, Cheng K (2009) Which portion of the natural panorama is used for view-based navigation in the Australian desert ant? J Comp Physiol A 195:681-689. https://doi.org/10.1007/s00359-0090443-6

Grieves RM, Jedidi-Ayoub S, Mishchanchuk K, Liu A, Renaudineau S, Jeffery KJ (2020) The placecell representation of volumetric space in rats. Nature Commun 11(1):1-13

Grobéty MC, Schenk F (1992) Spatial learning in a three-dimensional maze. Anim Behav 43(6):10111020

Hayman R, Verriotis MA, Jovalekic A, Fenton AA, Jeffery KJ (2011) Anisotropic encoding of threedimensional space by place cells and grid cells. Nat Neurosci 14:1182-1188. https://doi.org/ 10.1038/nn.2892

Hebets EA (2002) Relating the unique sensory system of amblypygids to the ecology and behavior of Phrynus parvulus from Costa Rica (Arachnida, Amblypygi). Can J Zool 80(2):286-295. https:// doi.org/10.1139/z02-006

Hebets EA, Aceves-Aparicio A, Aguilar-Argüello S, Bingman VP, Escalante I, Gering EJ, Nelson DR, Rivera J, Sánchez-Ruiz JÁ, Segura-Hernández L, Settepani V, Wiegmann DD, Stafstrom JA (2014a) Multimodal sensory reliance in the nocturnal homing of the amblypygid Phrynus pseudoparvulus (Class Arachnida, Order Amblypygi). Behav Proc 108(2014):123-130. https://doi.org/10.1016/j.be proc.2014.09.014

Hebets EA, Chapman RF (2000) Electrophysiological studies of olfaction in the whip spider Phrynus parvulus (Arachnida, Amblypygi). J Insect Physiol 46(11):1441-1448. https://doi.org/10.1016/S00221910(00)00068-8

Hebets EA, Gering EJ, Bingman VP, Wiegmann DD (2014b) Nocturnal homing in the tropical amblypygid Phrynus pseudoparvulus (Class Arachnida, Order Amblypygi). Anim Cogn 17(4):10131018. https://doi.org/10.1007/s10071-013-0718-8

Holbrook RI, de Perera TB (2009) Separate encoding of vertical and horizontal components of space during orientation in fish. Anim Behav 78(2):241-245

Holbrook RI, de Perera TB (2013) Three-dimensional spatial cognition: freely swimming fish accurately learn and remember metric information in a volume. Anim behav 86(5):1077-1083

Hölldobler B, Wilson EO (1990) The ants. Belknap, Cambridge Hurly TA, Franz S, Healy SD (2010) Do rufous hummingbirds (Selasphorus rufus) use visual beacons? Anim Cogn 13(2):377-383

Jeffery KJ, Jovalekic A, Verriotis M, Hayman R (2013) Navigating in a three-dimensional world. Behav Brain Sci 36(5):523-543

Jeffery KJ, Wilson JJ, Casali G, Hayman RM (2015) Neural encoding of large-scale three-dimensional space-properties and constraints. Front Psychol 6(July):1-12. https://doi.org/10.3389/fpsyg2015 00927 
Jovalekic A, Hayman R, Becares N, Reid H, Thomas G, Wilson J, Jeffery K (2011) Horizontal biases in rats' use of three-dimensional space. Behav Brain Res 222(2):279-288. https://doi.org/10.1016/ jbbr201102035

Nardi D, Newcombe NS, Shipley TF (2011) The world is not flat: can people reorient using slope? J Exp Psychol 37(2):354-367. https://doi.org/10.1037/a0021614

Nieh JC, Contrera FA, Ramírez Imperatriz-Fonseca SVL (2003) Variation in the ability to communicate three-dimensional resource location by stingless bees from different habitats. Anim Behav 66(6):1129-1139

Rheinlaender J, Hartbauer M, Römer H (2007) Spatial orientation in the bushcricket leptophyes punctatissima (phaneropterinae; orthoptera): I. phonotaxis to elevated and depressed sound sources. J Comp Physiol A 193(3):313-320. https://doi.org/10.1007/s00359-006-0186-6

Ronacher B (2020) Path integration in a three-dimensional world: the case of desert ants. J Comp Physiol A 206:379-387. https://doi.org/10.1007/s00359-020-01401-1

Santer RD, Hebets EA (2009) Tactile learning by a whip spider, Phrynus marginemaculatus CL Koch (Arachnida, Amblypygi). J Comp Physiol A 195(4):393-399. https://doi.org/10.1007/s00359-0090417-8

Segovia JMG, Neco LC, Willemart RH (2018) On the habitat use of the neotropical whip spider Charinus asturius (Arachnida: Amblypygi). Zool 35:1-6. https://doi.org/10.3897/zoologia35e12874

Steck K, Hansson BS, Knaden M (2009) Smells like home: Desert ants, Cataglyphis fortis, use olfactory landmarks to pinpoint the nest. Front Zool 6(1):5. https://doi.org/10.1186/1742-9994-6-5

Sumiya M, Fujioka E, Motoi K, Kondo M, Hiryu S (2017) Coordinated control of acoustical field of view and flight in three-dimensional space for consecutive capture by echolocating bats during natural foraging. PLoS ONE 12(1):e0169995

Ulanovsky N (2011) Neuroscience: How is three-dimensional space encoded in the brain. Curr Biol 21(21):R886-R888. https://doi.org/10.1016/j.cub.2011.09.031

Wehner R (2003) Desert ant navigation: how miniature brains solve complex tasks. J Comp Physiol A 189(8):579-588. https://doi.org/10.1007/s00359-003-0431-1

Weygoldt P (2000) Whip Spiders (Chelicerata: Amblypygi): their biology, morphology and systematics. Apollo Books, Stenstrup

Wiegmann DD, Moore CH, Flesher NR, Harper ED, Keto KR, Hebets EA, Bingman VP (2019) Nocturnal navigation by whip spiders: antenniform legs mediate near-distance olfactory localization of a shelter. Anim Behav 149:45-54. https://doi.org/10.1016/j.anbehav.2019.01.005

Yartsev MM, Ulanovsky N (2013) Representation of three-dimensional space in the hippocampus of flying bats. Science 340(6130):367-372

Zwergal A, Schöberl F, Xiong G, Pradhan C, Covic A, Werner P, Dieterich M (2016) Anisotropy of human horizontal and vertical navigation in real space: behavioral and PET correlates. Cereb Cortex 26(11):4392-4404 